Globe

Revue internationale d'études québécoises

\title{
Circulation des savoirs, mobilité internationale et études supérieures. Récit de la mise en place d'une voie favorisant l'insertion universitaire en milieu francophone nord-américain \\ Circulation of Knowledge, International Mobility and Higher Education. Supporting Integration at Francophone North-American Universities
}

\section{Marie-Claude Bernard}

Volume 17, numéro 2, 2014

Les enjeux du modèle des sociétés du savoir tels qu'ils sont vécus au Québec

URI : https://id.erudit.org/iderudit/1036239ar

DOI : https://doi.org/10.7202/1036239ar

Aller au sommaire du numéro

Éditeur(s)

Globe, Revue internationale d'études québécoises

ISSN

1481-5869 (imprimé)

1923-8231 (numérique)

Découvrir la revue

Citer cet article

Bernard, M.-C. (2014). Circulation des savoirs, mobilité internationale et études supérieures. Récit de la mise en place d'une voie favorisant l'insertion universitaire en milieu francophone nord-américain. Globe, 17(2), 93-115. https://doi.org/10.7202/1036239ar
Résumé de l'article

Dans un contexte de mobilité internationale, certaines universités recrutent un plus grand nombre d'étudiants et étudiantes en provenance de l'étranger. À la priorité de recrutement s'ajoutent alors les mesures d'accueil et de soutien à la réussite scolaire des nouveaux inscrits lors de leur première année du premier cycle, considérée comme cruciale dans leur parcours universitaire. Parmi les diverses stratégies de soutien déjà à l'oeuvre dans une université canadienne francophone, il est ici question d'un dispositif visant à favoriser leur insertion universitaire : est conçu un cours qui, selon une modalité d'enseignement interactive, vise à surmonter le sentiment d'étrangeté. Il propose une voie favorisant la compréhension des interventions et des outils pédagogiques et la réflexion sur les enjeux des représentations sociales et des stéréotypes culturels. Les étudiants sont amenés à socialiser entre eux tout en construisant leur identité étudiante et leur affiliation universitaire. Ils sont invités à examiner leurs rapports aux savoirs dans un contexte francophone nord-américain qui, selon une approche de formation pragmatique, confère pour d'aucuns un statut utilitaire aux savoirs. Cet article présente le dispositif en décrivant le contexte de mobilité internationale et en identifiant la problématique de la réussite des étudiants universitaires au premier cycle. Cette identification s'arrime avec les besoins et les compétences à développer par les nouveaux inscrits et s'articule par la suite avec le contenu du cours et son approche pédagogique. Dans la discussion, les considérations d'ordre pédagogique s'ouvrent vers celles de l'ordre de la communication interculturelle et soulèvent la question de l'importance des mesures et des stratégies de suivi qui doivent accompagner la mobilité internationale dans le milieu universitaire. 


\section{CIRCULATION DES \\ SAVOIRS, MOBILITÉ \\ INTERNATIONALE}

ET ÉTUDES SUPÉRIEURES. RÉCIT DE LA MISE EN

PLACE D'UNE VOIE

FAVORISANT L'INSERTION UNIVERSITAIRE

\section{EN MILIEU FRANCOPHONE NORD-AMÉRICAIN ${ }^{1}$}

\section{MARIE-CLAUDE BERNARD}

Université Laval

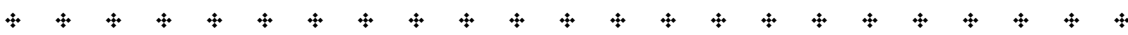

Résumé - Dans un contexte de mobilité internationale, certaines universités recrutent un plus grand nombre d'étudiants et étudiantes en provenance de l'étranger. À la priorité de recrutement s'ajoutent alors les mesures d'accueil et de soutien à la réussite scolaire des nouveaux inscrits lors de leur première année du premier cycle, considérée comme cruciale dans leur parcours universitaire. Parmi les diverses stratégies de soutien déjà à l'œuvre dans une université canadienne francophone, il est ici question d'un dispositif visant à favoriser leur

$$
\div+\div
$$

1. Je tiens à remercier Nicole Lacasse, Joanne Morneau ainsi que Sylvie Turcotte. Les deux premières œuvrent au vice-rectorat aux études et aux activités internationales de l'Université Laval. Leur travail concernant les mesures prises par le vice-rectorat pour le recrutement a été présenté dans une première version de ce travail lors d'une communication à la $5^{\text {th }}$ European First Year Experience Conference (2010). La troisième, en tant qu'adjointe à la direction de la Direction générale des programmes de premier cycle (DGPC), a rendu possible, dans les années 2009-2011, la préparation du cours «L'insertion universitaire en milieu francophone nord-américain ", sa mise en œuvre et sa diffusion aux différentes facultés de l'Université Laval. 
insertion universitaire : est conçu un cours qui, selon une modalité d'enseignement interactive, vise à surmonter le sentiment d'étrangeté. Il propose une voie favorisant la compréhension des interventions et des outils pédagogiques et la réflexion sur les enjeux des représentations sociales et des stéréotypes culturels. Les étudiants sont amenés à socialiser entre eux tout en construisant leur identité étudiante et leur affiliation universitaire. Ils sont invités à examiner leurs rapports aux savoirs dans un contexte francophone nord-américain qui, selon une approche de formation pragmatique, confère pour d'aucuns un statut utilitaire aux savoirs. Cet article présente le dispositif en décrivant le contexte de mobilité internationale et en identifiant la problématique de la réussite des étudiants universitaires au premier cycle. Cette identification s'arrime avec les besoins et les compétences à développer par les nouveaux inscrits et s'articule par la suite avec le contenu du cours et son approche pédagogique. Dans la discussion, les considérations d'ordre pédagogique s'ouvrent vers celles de l'ordre de la communication interculturelle et soulèvent la question de l'importance des mesures et des stratégies de suivi qui doivent accompagner la mobilité internationale dans le milieu universitaire.

$$
\div \quad \div
$$

\section{Circulation of Knowledge, International Mobility and Higher Education. \\ Supporting Integration at Francophone North-American Universities}

Abstract - In a global context of mobility, some universities are recruiting increasing numbers of international students. As a result, universities have focused their efforts not only on recruitment but also on supporting the academic success of new student during their first year as undergraduates, a crucial moment in their academic career. In addition to various support strategies already in place at one Canadian francophone university, this article will explore a measure intended to encourage student integration at the institution: an interactive course developed to overcome feelings of being an outsider. The course is intended to foster a better understanding of certain interventions and pedagogical tools, as well as reflection on issues surrounding social representations and cultural stereotypes. Students are invited to interact with one another as they as they develop their student identity and university affiliation. They are also encouraged to reflect on their relationship with knowledge in a francophone and North-American context, a context that favours a pragmatic approach to learning and a utilitarian conception of knowledge. The article explores the university's strategy by placing it in the context of international mobility and by highlighting the issue of undergraduate success. This question is closely related to that of new student's needs and the skills they should develop, concerns that are reflected in course content and pedagogical approaches. In turn, pedagogical considerations lead to a discussion of intercultural communication and raise the question of the importance of follow-up measures and strategies to support international mobility in a university environment.

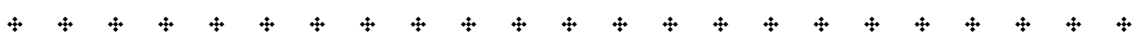

Mue par son idéal fondateur de former un espace public du savoir, l'UNESCO a intitulé son rapport de 2005 Vers les sociétés du savoir. À 
cette notion de "sociétés du savoir» se greffe l'objectif de l' "éducation pour tous tout au long de la vie", qui cherche à ne pas restreindre la question des savoirs aux avancées technologiques dans le domaine de l'information, mais à inclure plus largement les dimensions sociales, éthiques et politiques du savoir et, dans l'utilisation du pluriel, la diversité culturelle et linguistique des peuples ${ }^{2}$.

Dans ce contexte, l'Université Laval, comme d'autres universités canadiennes et nord-américaines, a développé une vision de l'internationalisation de ses activités universitaires. Des stratégies ont été mises en œuvre afin de créer et d'animer un environnement propice aux échanges interculturels, d'accroître le nombre d'étudiants d'origine étrangère, c'est-à-dire non canadiens (15\% dans le projet Horizon 2012) ${ }^{3}$, puis d'intensifier et de diversifier les programmes de mobilité internationale des étudiants (projet Horizon 2017) ${ }^{4}$.

L'accueil des étudiants et étudiantes d'origine étrangère pose la question de leur intégration et de leur encadrement. C'est pourquoi la Direction générale des programmes de premier cycle (DGPC) ${ }^{5}$ a commandé, au début des années 2000, une étude sur les manières de favoriser la réussite des étudiants d'origine étrangère, dont le taux de diplomation au premier cycle était plus bas que celui de leurs pairs québécois ${ }^{6}$. Aussi, à la demande du rectorat, un avis sur l'accueil, l'encadrement et l'intégration des étudiants étrangers a été publié 7 . À la suite de ces travaux, une réflexion a été conduite par la DGPC au sujet de la préparation des étudiants et étudiantes d'origine étrangère à intégrer les programmes de l'Université Laval et à réussir leurs

$$
\div+\div
$$

2. UNESCO, Vers les sociétés du savoir, Paris, Éditions de l'UNESCO, 2005. Néanmoins, derrière cette appellation de "sociétés du savoir " qui, dans le discours de l'organisation, vise à garantir le partage du savoir, l'ombre de la marchandisation demeure, et la représentation des savoirs comme une sorte de carte de distribution de richesse et de puissance est présente et mérite qu'on y prête attention.

3. Université LAVAL, "Horizon 2012. Orientations de développement de l'Université Laval », http:// www2.ulaval.ca/fileadmin/cabinetrecteur/brochure_horizon2012_3.pdf (15 février 2014).

4. UNIVERSITÉ LAVAL, "Horizon 2017. Orientations de développement de l'Université Laval», http://www2.ulaval.ca/fileadmin/cabinetrecteur/horizon-2017-universite-laval.pdf (15 février 2014).

5. La Direction générale des programmes de premier cycle est mandatée, entre autres, pour donner les cours compensateurs et coordonner l'ensemble des programmes du premier cycle, ce qui inclut notamment l'harmonisation des exigences des programmes. Elle veille à la qualité de la formation ainsi qu'à l'encadrement dans les programmes de premier cycle.

6. Par contre, le taux de diplomation aux études de $2^{\mathrm{e}}$ et $3^{\mathrm{e}}$ cycles chez les étudiants étrangers était supérieur à celui des étudiants québécois. Le suivi du taux de diplomation au $1^{\text {er }}$ cycle des cohortes allant de 2000 à 2009 est présenté plus loin.

7. COMMISSION DES AFFAIRES ÉTUDIANTES, «Avis sur l'accueil, l'encadrement et l'intégration des étudiants étrangers à l'Université Laval», document non publié, Université Laval, 2006 et Annie PILOTE, «Favoriser la réussite des étudiants d'origine étrangère à l'Université Laval. Analyse de besoins dans une démarche de conception d'une formation ", rapport non publié, Université Laval, 2004. 
études. Ce qui leur était alors offert, à savoir des cours (parfois obligatoires) visant la connaissance du contexte d'accueil, l'application de techniques métacognitives et l'application de méthodes d'études ou, encore, des cours dits " compensateurs» dispensés dans le but de les préparer à atteindre les prérequis de leur programme, ne semblait plus suffisant pour assurer la réussite de leur cursus.

Cet article a pour but de présenter un des dispositifs mis en œuvre par l'Université Laval dans la foulée de cette réflexion : le cours «L'insertion universitaire en milieu francophone nord-américain ", destiné aux étudiantes et étudiants étrangers inscrits dans un programme de premier cycle. Ce cours constitue une intervention pédagogique qui, tout en ayant pour but d'aider les étudiants et étudiantes à comprendre la démarche d'étude en vigueur à l'Université Laval, est aussi une expérience visant à construire une société du savoir au Québec. Le cours et sa création sont présentés sous une forme narrative. Cette présentation est précédée d'une brève mise en contexte de la mobilité internationale favorisée par les études supérieures et suivie d'une discussion sur la pertinence de cet enseignement.

\section{MOBILITÉ INTERNATIONALE ET ÉTUDES SUPÉRIEURES}

À l'échelle mondiale, le flux d'étudiantes et étudiants étrangers a connu une augmentation depuis la fin des années 1970. Des données de l'OCDE combinées à celles de l'Institut de statistique de l'UNESCO révèlent une forte croissance des effectifs étudiants en formation à l'étranger, qui aurait quadruplé de 1975 à $2009^{8}$. Selon l'OCDE, le nombre d'étudiantes et d'étudiants étrangers dans l'enseignement supérieur a progressé de $77 \%$ dans le monde depuis l'an 2000. Les pays européens représentent les pays d'accueil les plus prisés $(38 \%)$, suivis des pays d'Amérique du Nord (23\%). Toujours selon l'OCDE, cette internationalisation serait le reflet d'activités énergiques de recrutement de la part des universités d'accueil, mais aussi de transformations économiques et sociales qui favorisent la mobilité et les liens politiques, sociaux, culturels et académiques entre les pays, l'accroissement des taux d'accès à l'enseignement supérieur ${ }^{9}$, ainsi que la démocratisation des prix du transport aérien.

$$
4+4
$$

8. OCDE, "Chapitre C. Accès à l'éducation, participation et progression ", Regards sur l'éducation. Les indicateurs de l'OCDE, http://www.oecd.org/fr/education/scolaire/regardssurleducation2011lesindicateurs delocde.htm (4 mars 2014).

9. Non seulement le taux d'étudiantes et étudiants étrangers a augmenté, mais les effectifs scolarisés dans l'enseignement supérieur ont progressé dans le monde de façon considérable depuis 2000 (65\%, selon les mêmes statistiques de l'OCDE, 2011). 
Au Canada, comme dans d'autres pays membres de l'OCDE, le nombre d'étudiants étrangers a plus que doublé depuis $2000^{10}$. Les cinq pays qui accueillent le plus d'étudiants et d'étudiantes de l'étranger (plus de la moitié) sont les États-Unis, le Royaume-Uni, l'Australie, l'Allemagne et la France. Le Canada arrive en sixième place avec $5 \%$ des effectifs, selon les chiffres de 2009 rapportés par l'OCDE ${ }^{11}$. D'après l'enquête de 2009 sur les étudiants étrangers réalisée pour le Bureau canadien de l'éducation internationale ${ }^{12}$, plus de 178000 étudiantes et étudiants étrangers étudiaient au Canada en 2008, et plus de la moitié, en 2005, venaient d'Asie. Les données plus récentes de Citoyenneté et Immigration Canada enregistrent un total de 265404 étudiantes et étudiants étrangers inscrits au Canada en 2012 ${ }^{13}$, dont 38114 (soit 14,4\%) dans la province de Québec ${ }^{14}$. Si la moitié des effectifs entrant au Canada pour faire des études viendraient d'Asie, cela n'est pas le cas de la province de Québec, qui enregistre des données différentes en raison, notamment, de la langue française qui est majoritairement la langue d'enseignement universitaire. Un des éléments importants dans le choix du pays d'accueil est celui de la langue d'enseignement ${ }^{15}$.

Dans la province de Québec, la progression du nombre d'étudiantes et étudiants étrangers se serait accélérée à partir des années 1995, atteignant $10 \%$ de croissance annuelle au cours des dernières années du $\mathrm{XX}^{\mathrm{e}}$ siècle $^{16}$. À l'Université Laval, cette croissance aurait débuté dès les années $1990^{17}$. Selon les données du bureau du registraire, le nombre d'étudiantes et d'étudiants internationaux est passé de 1968 à l'automne 2000, à 2292 en 2005, à 2536 à l'automne 2010 et à 3250 à l'automne 2013, soit respectivement une augmentation de $16 \%$, de $11 \%$ et de $28 \%$.

$$
\div+\div
$$

10. OCDE, "Chapitre C. Accès à l'éducation, participation et progression », op. cit., p. 345.

11. Ibidem.

12. Le Bureau canadien de l'éducation internationale (BCEI) a réalisé ce même type d'enquête en 1999 , en 2004 et en 2009. Pour la plus récente, voir Jennifer HuMPHRIES et Janine KNIGHT-GrOFE, «Le Canada au premier rang. L'enquête de 2009 sur les étudiants étrangers », http://www.cbie-bcei.ca/wpcontent/uploads/2013/03/Le-Canada-au-premier-rang-2009.pdf (4 mars 2014).

13. Citoyenneté et Immigration CANADA, "Canada. Faits et chiffres. Aperçu de l'immigration. Résidents permanents et temporaires 2012 ", Effectif au $1^{\text {er }}$ décembre des étudiants étrangers selon les pays d'origine, p. 86, http://www.cic.gc.ca/francais/ressources/statistiques/menu-faits.asp (4 mars 2014).

14. Ibid., p. 89-90.

15. OCDE, «Chapitre C. Accès à l'éducation, participation et progression », op. cit., p. 347.

16. Nicole LACASSE, "Clés de l'internationalisation II. Mieux connaître et satisfaire les étudiants universitaires internationaux. Un investissement d'avenir", Québec, Université Laval, Faculté des sciences de l'administration, Chaire Stephen Jarislowsky en gestion des affaires internationales, 2005.

17. En 1995, une politique d'accueil des étudiants étrangers a été mise en place; elle sera abrogée en 2001 au profit d'une politique ciblant l'ensemble des étudiants de l'Université Laval (voir COMMISSION DES AFFAIRES ÉTUDIANTES, «Avis sur l'accueil, l'encadrement et l'intégration des étudiants étrangers à l'Université Laval», op. cit., p. 9). 
L'Université Laval est une université francophone nordaméricaine qui a signé de nombreuses ententes internationales avec plus de 500 partenaires établis dans plus de 69 pays. Sa population étudiante provenant de l'étranger se démarque de celles d'autres provinces canadiennes, puisqu'elle est majoritairement d'Europe et d'Afrique. Cette université accueille peu d'étudiants et d'étudiantes en provenance de la région AsieMoyen-Orient-Océanie et un très faible pourcentage issu des États-Unis. En 2013-2014, la provenance de 3250 étudiantes et étudiants étrangers se répartit ainsi, selon les données du bureau du registraire résumées dans le tableau 1:

TABLEAU 1

RÉPARTITION DES ÉTUDIANTES ET ÉTUDIANTS D'ORIGINE ÉTRANGÈRE À L'UNIVERSITÉ LAVAL PAR RÉGION 2013-2014

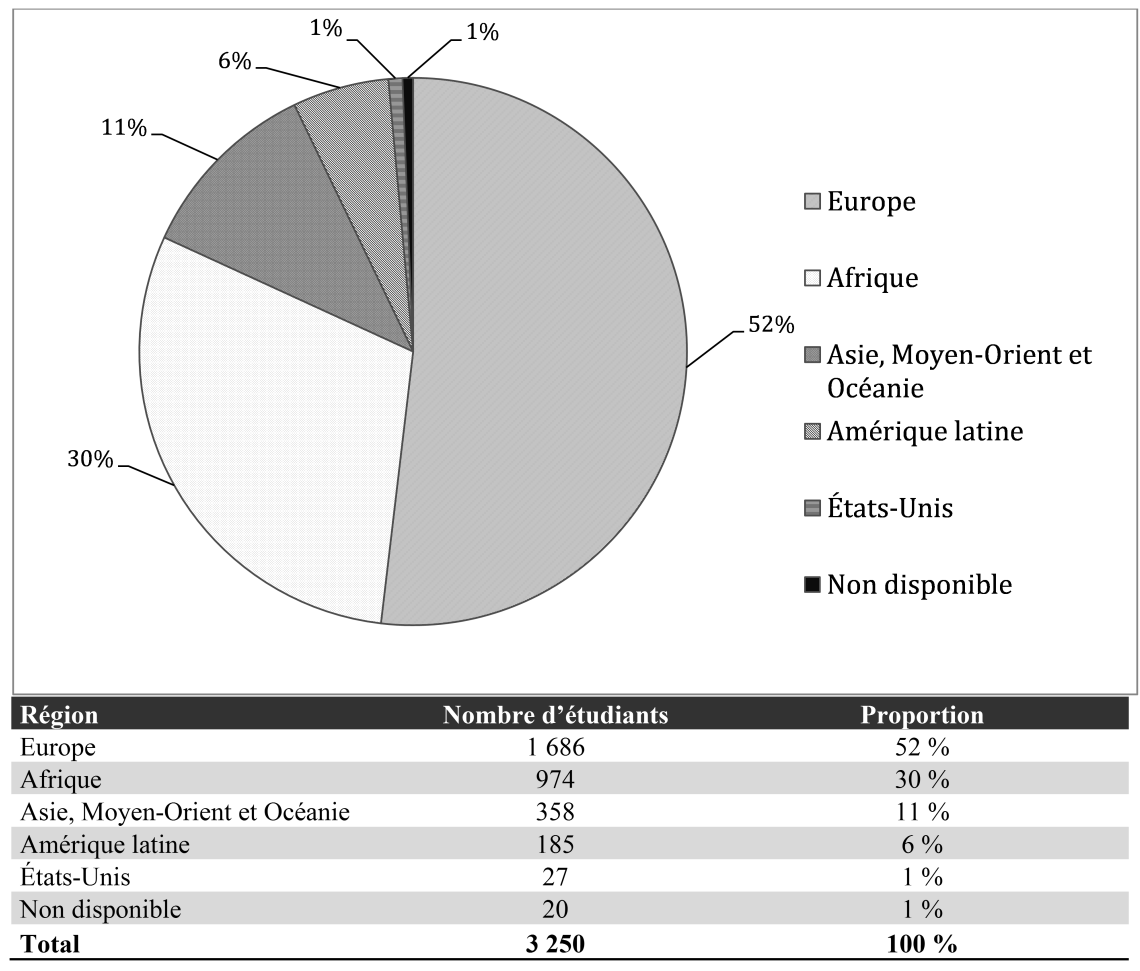


En pourcentage, toujours selon les statistiques du bureau du registraire, les dix pays étrangers les plus représentés sur le campus en 2013-2014 sont la France, le Maroc, la Tunisie, la Chine, l'Iran, la Côte d'Ivoire, le Sénégal, le Cameroun, le Bénin et l'Allemagne (voir tableau 2).

\section{TABLEAU 2}

RÉPARTITION DES ÉTUDIANTES ET ÉTUDIANTS D'ORIGINE ÉTRANGÈRE PAR PAYS DE PROVENANCE À L'UNIVERSITÉ LAVAL (2013-2014)

\begin{tabular}{|l|}
\hline \\
\end{tabular}


La France est largement le pays au taux le plus élevé de provenance des étudiants et étudiantes de l'étranger. Six pays africains francophones figurent parmi les 10 principaux pays d'origine des étudiants internationaux ${ }^{18}$.

En ce qui concerne le cycle d'études visé, ce sont les étudiants du premier cycle qui forment le contingent le plus important d'étudiantes et étudiants étrangers inscrits à l'Université Laval, selon le bureau du registraire (voir tableau 3).

\section{TABLEAU 3}

RÉPARTITION DES ÉTUDIANTES ET ÉTUDIANTS D'ORIGINE ÉTRANGÈRE PAR CYCLE D'ÉTUDES INSCRITS À L'UNIVERSITÉ LAVAL (2013-2014)

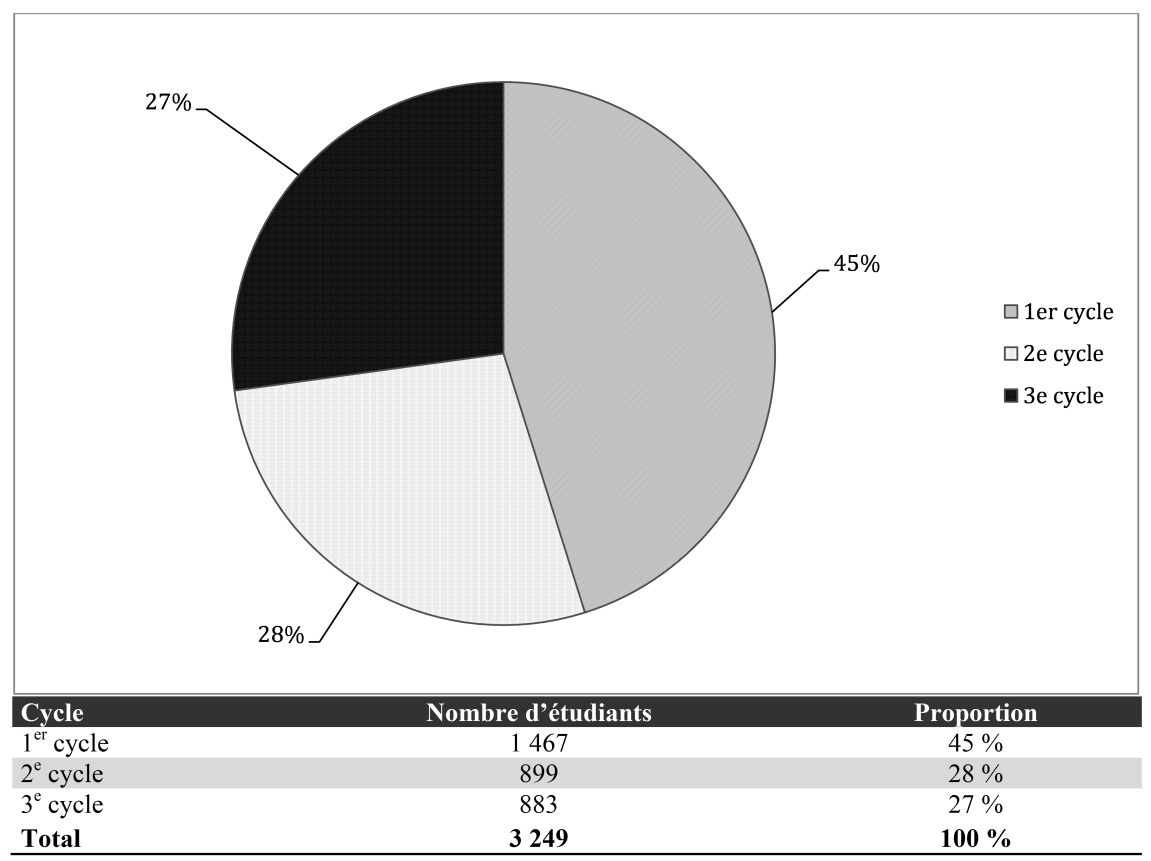

Regardons maintenant comment se répartissent les étudiants et étudiantes de premier cycle selon leur pays d'origine. Les données du tableau 4 ont été obtenues des statistiques du bureau du registraire sur les inscriptions $\mathrm{du}$

$$
\div+\div
$$

18. Le Mexique est le seul pays d'Amérique latine qui s'est trouvé parmi les 10 principaux pays d'origine (année 2010-2011), mais le taux d'étudiantes et étudiants en provenance de ce pays a baissé depuis. Alors qu'il était de 46 sur 2536 (1,81\%) en 2010-2011, le taux d'étudiants en provenance de ce pays est, en 2013-2014, de 18 sur 3250 (0,55\%), ce qui représente une variation de -0,69\%. 
premier cycle pendant l'année 2010-2011 en ne retenant que les 10 premiers pays représentés selon le nombre d'inscriptions.

\section{TABLEAU 4}

RÉPARTITION DES ÉTUDIANTES ET ÉTUDIANTS D'ORIGINE ÉTRANGÈRE INSCRITS AU PREMIER CYCLE À L'UNIVERSITÉ LAVAL SELON LES 10 PREMIERS PAYS DE PROVENANCE (2010-2011) ${ }^{19}$

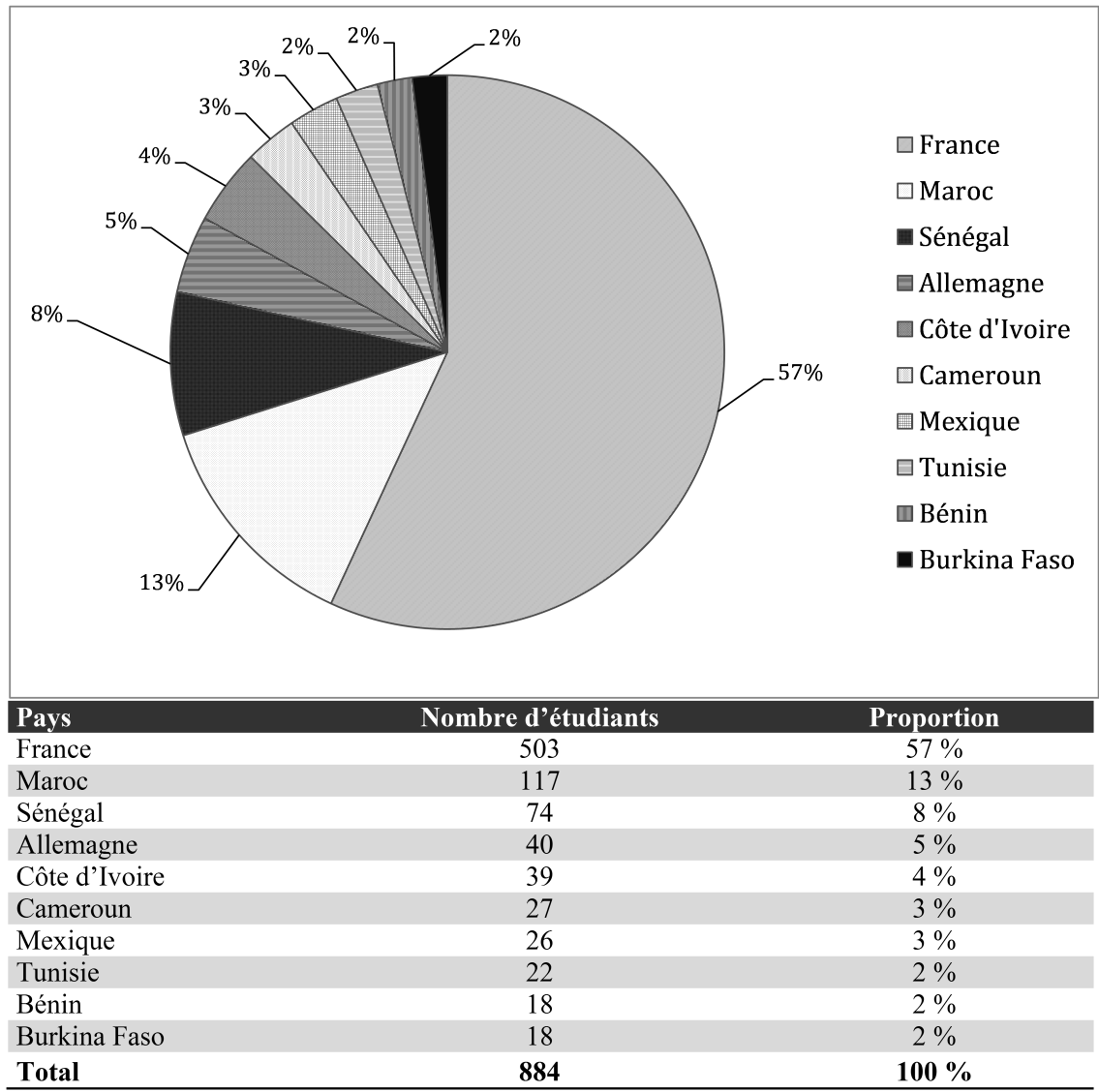

Si l'on compare ces statistiques à celles du tableau 2, qui inclut tous les étudiantes et étudiants étrangers inscrits, on remarque quelques différences.

$$
\div+\div
$$

19. Les premières cohortes du cours reflétaient, à quelques exceptions près, les pays représentés dans ce tableau, en ajoutant trois autres pays d'Afrique subsaharienne francophone. 
Ainsi, le Mexique et le Burkina Faso se trouvent dans les dix premiers pays représentés au premier cycle, mais n'apparaissent pas dans les dix premières places pour les trois cycles. A contrario, l'Iran et la Chine se trouvent dans le classement général, mais ne se trouvent pas parmi les dix principaux pays d'origine des étudiantes et étudiants étrangers inscrits au premier cycle de cette année scolaire. Parmi les dix pays du tableau 4, huit sont des pays francophones, ce qui confirme l'importance de la langue dans le choix de l'Université pour les étudiants et étudiantes d'origine étrangère.

\section{LE DÉFI DE LA PREMIÈRE ANNÉE DU PREMIER CYCLE}

La priorité de mettre en œuvre des dispositifs d'accueil et d'aide à la réussite scolaire à l'université, particulièrement au premier cycle, est de plus en plus présente dans plusieurs pays. L'Université Laval n'est pas l'exception : depuis plusieurs années, elle manifeste un souci pour la réussite scolaire de ses étudiants et étudiantes. Or l'analyse des statistiques des inscriptions et du taux de diplomation montrait un décalage entre le taux de réussite des étudiantes et étudiants étrangers et celui de leurs pairs québécois. Plus précisément, le suivi statistique des cohortes étudiantes au premier cycle des années 2000 à 2009 indique un taux de diplomation, pour les populations étudiantes d'origine québécoise et canadienne, de $78 \%$, alors qu'il est de $59 \%$ chez les étudiantes et étudiants ayant un permis de séjour ou de résidents permanents (ces deux statuts concernent particulièrement les effectifs en provenance de l'étranger ${ }^{20}$. En vue de favoriser la réussite sociale et scolaire des nouveaux étudiants et étudiantes de l'international et de soutenir leur cheminement étudiant, l'Université Laval a mis en place plusieurs mesures. Parmi celles-ci, nous comptons des actions conduites par le Bureau d'accueil des étudiants étrangers $(\mathrm{BAEE})^{21}$, telles que le soutien à la recherche d'un logement en dehors du campus pour les nouveaux arrivants ou le programme de jumelage des étudiants étrangers. De plus, le service des résidences favorise le travail rémunéré dans les résidences universitaires et organise avec le Bureau de la vie étudiante (BVE) des activités culturelles sur le campus et des visites hors campus. Le Centre d'aide aux étudiants soutient également les étudiants et étudiantes en offrant des cours concernant notamment l'appropriation de stratégies d'apprentissage, comme la gestion du temps, ou les façons de surmonter des difficultés d’apprentissage telles que le

$$
4+4
$$

20. Le suivi des cohortes, réalisé à partir des données du système d'information sur les études, a été préparé par Luc Simon (10 février 2015) et compilé par le Bureau de planification et d'études institutionnelles. 21. Il porte à présent le nom de Bureau de la vie étudiante (BVE). 
stress ou l'anxiété scolaire. Dans le cadre des cours "compensateurs ", la DGPC propose par exemple les cours "Apprentissage autonome ", "Découvrir le Québec", "Introduction à l'argumentation » ou "Dissertation historique ".

En prolongeant la réflexion, les responsables ont eu l'idée d'ajouter à ces mesures une intervention pédagogique qui pourrait agir de manière préventive, c'est-à-dire en limitant le taux d'échec. Chargée de mettre ce cours en place, j'ai commencé par faire une revue des travaux portant sur les principales difficultés des étudiantes et étudiants étrangers arrivant dans un programme de premier cycle. J'ai pu constater que la première année universitaire est cruciale pour tous les étudiantes et étudiants ${ }^{22}$, comme le montrent les travaux sur la "First Year Experience» (FYE), qui s’intéressent à différentes facettes de l'intégration au milieu universitaire ${ }^{23}$. Parmi les difficultés relevées figurent la découverte d'un nouveau milieu institutionnel, l'apprentissage d'un travail de conceptualisation différent de celui qui caractérise les études secondaires, l'organisation de l'emploi du temps et des moyens nécessaires pour répondre aux exigences du travail universitaire ${ }^{24}$. Certains travaux considèrent cette première année universitaire comme une étape de transition pendant laquelle de nouvelles stratégies d'adaptation sont requises, autant en ce qui concerne les façons d'approcher les études que les dispositions sociales permettant d'établir de nouvelles relations ${ }^{25}$.

Sous un angle plus sociologique, d'autres travaux ont étudié l'organisation pédagogique, le poids du nombre d'étudiants et d'étudiantes dans les classes, ainsi que leur diversité (hétérogénéité). Ces phénomènes découlent de la "massification " des études universitaires à la suite de l'accroissement des taux d'accès à l'enseignement supérieur qui a donné lieu au développement de stratégies de soutien offertes par les universités d'accueil ${ }^{26}$. En général, ces stratégies incluent des activités d'orientation, de soutien

$$
4+4
$$

22. Alain COULON et Saeed PAIVANDI, État des savoirs sur les relations entre les étudiants, les enseignants et les IATOSS dans les établissements d'enseignement supérieur, Paris, Observatoire de la vie étudiante (OVE), 2008.

23. Craig MCINNIS, «Researching the First Year Experience. Where to from Here? ", Higher Education Research \& Development, vol. 20, n ${ }^{\circ}$ 2, 2001, p. 105-114.

24. Joe CUSEO, "The "Empirical" Case for the First-Year Seminar. Promoting Positive Student Out-comes and Campus-Wide Benefits", The First-Year Seminar. Research-Based Recommendations for Course Design, Delivery, \& Assessment, Dubuque, IA, Kendall/Hunt, 2010.

25. Mark PeEl, Stephen POWELl et Mia TREACEY, "Student Perspectives on Temporary and Permanent Exit from University. A Case Study form Monash Universtiy", Journal of Higher Education Policy and Management, vol. 26, $\mathrm{n}^{\circ}$ 2, 2004, p. 239-249.

26. À titre d'exemple, voir L'AGENCE FRANÇAISE POUR LA PROMOTION DE L'ENSEIGNEMENT SUPÉRIEUR, L'ACCUEIL ET LA MOBILITÉ INTERNATIONALE, "Les notes Campus France», http://www. campusfrance.org (13 mai 2015). 
psychologique, d'aide en "méthodes de travail " ou en "méthodes d'apprentissage " (gestion du temps, démarches de recherche documentaire, techniques de mémorisation, guides de préparation aux examens, par exemple), ou encore l'offre d'outils ou de moyens qui favoriseraient l'établissement d'un dialogue aisé avec les pairs. Dans cette optique, des approches collaboratives sont encouragées, tels les programmes de parrainage, le mentorat et le coaching entre étudiants. Inspirés de la psychologie humaniste, plusieurs travaux indiquent l'importance de l'image de soi des étudiants et étudiantes et de la reconnaissance de leur potentiel ${ }^{27}$. D'autres programmes d'appui soulignent l'importance d'outiller les étudiants et étudiantes en matière de nouvelles technologies $\mathrm{Web}^{28}$. Romainville insiste sur le rôle de l'enseignement et plaide pour le développement d'une pédagogie universitaire qui tienne compte du contexte ${ }^{29}$.

En sociologie de l'éducation, les travaux de Coulon ont mis en relief que, pour réussir aux études supérieures, il faut apprendre le "métier" d'étudiant. En effet, le statut de l'étudiant au secondaire (lycéen en France) n'est pas celui de l'étudiant universitaire ; une transformation progressive du lycéen en étudiant est nécessaire dans la réussite des études universitaires. Coulon distingue trois phases dans cet apprentissage : le "temps de l'étrangeté ", le "temps de l'apprentissage" et le "temps de l'affiliation ». À cette dernière phase, l'étudiant ou l'étudiante a acquis une certaine maîtrise de l'interprétation et de l'usage des règles universitaires (affiliation institutionnelle) pouvant aller jusqu'à l'affiliation intellectuelle, qui inclut la compréhension des sous-cultures caractérisant le champ d'études particulier dans lequel l'étudiant ou l'étudiante en question se situe ${ }^{30}$. Les travaux situés dans la lignée des approches interculturelles sont également pertinents, notamment ceux qui concernent l'analyse des représentations sociales et du langage interculturel ${ }^{31}$.

$$
\div \div
$$

27. Barbara FREDRICKSON et Thomas JOINER, «Positive Emotions Trigger Upward Spirals toward Emotional Well-being", Psychological science, vol. 13, n 2, 2002, p. 172-175.

28. Cet enjeu est majeur lorsqu'on considère l'expansion de l'utilisation des outils informatiques et de la diffusion des savoirs à travers le Web.

29. Marc Romainville, L'échec dans l'université de masse, Paris, L'Harmattan, 2000 et "L'évaluation de la qualité de l'enseignement dans le supérieur", Enseignement supérieur en Europe, vol. 24, n 3, 1999, p. 117-130.

30. Alain Coulon, Le métier d'étudiant. L'entrée dans la vie universitaire, Paris, Economica-Anthropos, 1997.

31. Pierre R. DASEN et Christiane PERREgaux (dir.), Pourquoi des approches interculturelles en sciences de l'éducation, Bruxelles, De Boeck Université, 2002 et Myriam GraBER, Claire-Lise MÉGARD MuTEZINTARE et Théogène-Octave GAKUBA, Les étudiants d'Afrique subsaharienne, Genève, IES éditions, 2010. Les travaux d'Abdallah-Pretceille prônent une éducation interculturelle et ceux de Fred Dervin 


\section{PROCESSUS DE CONSTITUTION DU COURS «INSERTION UNIVERSITAIRE EN MILIEU FRANCOPHONE NORD-AMÉRICAIN ${ }^{32}$ "}

Après la recherche documentaire décrite ci-dessus, j'ai examiné des cours de premier cycle offerts à l'Université Laval qui pouvaient se rapprocher de mon objectif: les cours dits "compensateurs" cités ci-dessus, ainsi que différents cours abordant d'une manière ou d'une autre la question de l'insertion des étudiantes et étudiants étrangers (par exemple, "Monde des affaires dans le contexte nord-américain ", "Le choc culturel à l'échelle internationale ", "Diversité culturelle: contextes et enjeux»). Cet examen s'est enrichi d'un tour d'horizon des activités, des stratégies ou des cours du même ordre proposés par d'autres universités ${ }^{33}$.

Parallèlement, des rencontres ont été planifiées avec différents intervenants de l'Université Laval, tels que les responsables des cours mentionnés ci-dessus, le responsable du Centre d'aide aux étudiants et ceux du Bureau de la vie étudiante ${ }^{34}$. Ces personnes partageaient d'une manière ou d'une autre l'objectif de la réussite scolaire des étudiants et étudiantes et avaient les mêmes sujets de préoccupation à cet égard. J'en cite quelquesuns : la maîtrise de la langue (et plus particulièrement le rapport à l'écriture et à la lecture), la formation antérieure, les notions de motivation et de persévérance dans les études, l'anxiété face aux études et aux méthodes de travail, le rythme des études et le rapport au temps, le décalage dans les compétences informatiques acquises préalablement, des problèmes de

$$
\div+\div
$$

incluent des propositions pour l'acquisition de compétences langagières et interculturelles. Voir Martine ABDAllah-PRETCEILle, L'éducation interculturelle, Paris, Presses universitaires de France, 1999 et Fred DERVIN et Eija SUOMELA-SALMI (dir.), Évaluer les compétences langagières et interculturelles dans l'enseignement supérieur, Turku, Département d'études françaises, 2007, p. 115-118.

32. Le travail de conception du cours s'est étalé de l'automne 2009 à l'hiver 2010. La première cohorte s'est inscrite à l'automne 2010. Depuis cette session-là, le cours est offert aux étudiantes et étudiants d'origine étrangère pendant les sessions d'automne et d'hiver. Il a subi quelques modifications à partir de l'automne 2011 lorsqu'il a été pris en charge par une autre chargée de cours et, à l'hiver 2015, il a été offert pour la première fois à distance. Les cohortes inscrites à l'automne, composées d'une vingtaine d'étudiants, sont plus nombreuses que celles de l'hiver, formées d'une dizaine d'étudiants.

33. Ce tour d'horizon inclut les universités au Québec, au Canada, en France et aux États-Unis. Pour ce dernier pays, ce fut le cas plus particulièrement de la University of South Carolina (USC), qui a donné naissance au courant FYE et qui, cherchant à favoriser l'intégration et la réussite universitaires, propose un séminaire de première année connu sous le titre "University 101 ".

34. Une autre rencontre a été prévue avec Nathalie Barbeau, dont l'expérience d'accueil et d'intégration auprès des étudiantes et étudiants étrangers à l'Université Laval et le mémoire de maîtrise furent également éclairants (Nathalie BARBEAU, «La situation des étudiants étrangers à l'Université Laval. Portrait de leur situation d'adaptation et d'intégration dans le contexte de l'institution d'enseignement, de la politique d'immigration Canada/Québec et de la société québécoise ", mémoire de maîtrise (service social), Québec, Université Laval, 2004). Par contre, il manque à la liste les associations étudiantes, que nous n'avons malheureusement pas contactées, ce qui constitue une limite dans le processus de constitution du cours. 
compréhension des attentes universitaires, des obstacles à l'insertion dans les travaux d'équipe, des difficultés à participer verbalement en cours, des lacunes dans l'appropriation de méthodes pour étudier, pour prendre des notes ou pour relever les idées importantes dans un texte, ou encore une compréhension divergente des consignes écrites, une interprétation différente du respect de la propriété intellectuelle et d'autres malentendus concernant, par exemple, ce qui est considéré (ou non) comme du plagiat.

L'étude documentaire, enrichie par ces échanges, a permis de tracer trois pistes de réflexion. La première touchait les difficultés rencontrées par les étudiantes et étudiants étrangers et leurs besoins, tels que décrits cidessus.

La deuxième concernait les attentes du système d'enseignement nord-américain québécois. En effet, cet ancrage culturel se distingue des systèmes francophones d'autres horizons, d'où provient la majorité des étudiantes et étudiants étrangers inscrits à l'Université Laval. Disons rapidement, ici, que le système québécois semble avoir opté pour une approche plutôt pragmatique, voire utilitaire et instrumentale, de la formation universitaire de premier cycle et de la transmission des savoirs qui s'y produit. Selon Laszlo ${ }^{35}$, pour les Nord-Américains, l'éducation représente avant tout un investissement qui cherche à être rentable. L'historien Jean Hamelin estime que l'université québécoise privilégie la formation d' "experts» et de "promoteurs de recherche» dans un cadre concurrentiel, plutôt que celle de «savants» ou de "penseurs scientifiques ${ }^{36}$. Lenoir associe également la formation universitaire québécoise à la logique nord-américaine, qu’il qualifie de néolibérale, pragmatique, utilitaire et instrumentale ${ }^{37}$. Partant de ce point de vue, j’ai dressé une liste de ce qui pourrait être qualifié de particularités des universités nord-américaines, telles que les travaux en équipe, l'importance du réseautage, les examens présentés sous forme de questions à choix multiple et l'apprentissage par problèmes ou présentant des mises en situation inspirées du milieu.

$$
\div+\div
$$

35. Dans un ouvrage concis, Pierre Laszlo explique les particularités des universités nord-américaines en situant leur enracinement dans un héritage britannique et germanique qui les distinguerait des universités européennes actuelles en ce qui concerne, par exemple, l'architecture (des campus), l'importance donnée au sport, les laboratoires et l'équipement de pointe, ainsi que le rapport aux collectivités locales (Pierre LASZLO, Les universités américaines, Paris, Flammarion, 1996).

36. Jean Hamelin, cité dans James LAMBERT, «Évolution et transformation d'une institution ", Cap-auxDiamants, $\mathrm{n}^{\circ}$ 72, 2003, p. 14.

37. Yves LENOIR, «Le "rapport Parent”, point de départ de l'ancrage de l'école québécoise dans la logique anglophone nord-américaine", Canadian Journal of Education/Revue canadienne de l'éducation, vol. 28, $\mathrm{n}^{\circ} 4$, 2005, p. 638-668. 
La troisième piste de réflexion tentait de cerner les thématiques à aborder dans un cours ayant, entre autres, la finalité de diminuer l'écart entre les besoins identifiés des étudiantes et étudiants non canadiens (voir première piste citée ci-dessus) et les attentes du système d'enseignement.

J'ai également comparé les besoins identifiés par la revue de littérature (service d'information, activités d'accueil et d'intégration, service d'orientation, conseils dans une perspective scolaire, adaptation aux méthodes d'apprentissage, communication interculturelle, aide en français, contrat d'engagement, mode d'évaluation, aspects culturels des savoirs, modes de recherche documentaire, compétences technologiques et informatiques, etc.) et les éléments déjà pris en charge par différentes instances de l'Université Laval, par exemple, les compétences technologiques et informatiques ${ }^{38}$ ou l'aide en français, ce qui m’a permis d'éliminer certains aspects. Par contre, d'autres éléments, soit des besoins qui n'étaient traités ni dans les cours ni dans d'autres interventions, ont été retenus.J'ai alors défini les objectifs du cours comme suit: aider les étudiants et étudiantes d'origine étrangère à s'approprier les outils pédagogiques propres à l'Université Laval et à comprendre le processus d'apprentissage. À ces deux objectifs s'est ajouté celui, inspiré des travaux de Coulon, de favoriser l'affiliation ${ }^{39}$ et l'engagement ${ }^{40}$ des étudiantes et étudiants étrangers. En effet, dans la conceptualisation du "métier d'étudiant" que propose cet auteur, qui a étudié les étudiantes et étudiants du premier cycle universitaire pour remédier aux échecs et aux abandons importants documentés dans la scolarisation de ce cycle en France ${ }^{41}$, l'affiliation et l'engagement seraient fortement liés à la réussite universitaire. L'affiliation renvoie à une phase que traversent les étudiantes et étudiants lorsqu'ils arrivent non seulement à surmonter le temps de l'étrangeté et à déchiffrer les codes institutionnels et intellectuels de leur

$$
4 \div 4
$$

38. De façon parallèle à l'élaboration de ce cours, un cours sur la réussite aux études universitaires à l'ère numérique a vu le jour à la Faculté des sciences de l'éducation.

39. Alain Coulon, Le métier d'étudiant..., op. cit.

40. Alexander C. MCCORMICK, Jillian KINZIE et Robert M. GONYEA, «Student Engagement. Bridging Research and Practice to Improve the Quality of Undergraduate Education ", Michael B. PAULSEN et John C. SMART (dir.), Higher Education. Handbook of Theory and Research, Dordrecht, Springer, 2013, p. 47-92.

41. Les travaux d'Alain Coulon sur l'échec et l'abandon scolaire dans le contexte de la réforme Savary et des premiers cycles à la fin du siècle en France, réalisés selon une approche ethnométhodologique, ont relevé plusieurs problèmes. Depuis, de nombreuses initiatives ont vu le jour et certains de ces problèmes ont été surmontés, malgré des moyens relativement faibles; par contre, d'autres sont apparus et le problème du décrochage à l'université reste prégnant (François SARFATI, "L'université face au décrochage», http://www.laviedesidees.fr/IMG/pdf/20150413_decrochage1-2.pdf (14 avril 2015). Par ailleurs, la voie d'analyse empruntée par Alain Coulon et sa conceptualisation du "métier d'étudiant " ainsi que des phases d'intégration aux études universitaires ont montré leur fécondité et demeurent actuelles. 
nouvel «habitus", mais aussi à assimiler les codes du savoir universitaire. L'engagement, en tant qu'action d'investissement personnel, favoriserait la persévérance scolaire.

Des entretiens individuels ont alors été menés, d'une part, auprès d'étudiantes et étudiants étrangers qui avaient réussi leur intégration et venaient d'obtenir leur diplôme (ou étaient en voie de l'obtenir) et, d'autre part, auprès d'étudiantes et étudiants étrangers qui, au contraire, étaient en situation d'échec (exclus de leur programme, ils essayaient une reprise en s'inscrivant aux études libres ou étaient en voie de décider leur retrait définitif de l'université). Recrutés par le Bureau de la vie étudiante et la DGPC, qui les avaient retenus en tant que volontaires à participer à des entrevues de suivi, cinq étudiants et étudiantes ont ainsi participé volontairement à des entretiens semi-dirigés tenus sous le mode de brefs récits de parcours; trois se trouvaient en situation de "réussite» et deux, en situation d' "échec».

Une première question leur proposait de raconter le parcours les ayant conduits à l'Université Laval. Ensuite, je leur demandais de me raconter la façon dont s'était déroulée leur arrivée à l'université : les liens sociaux, les questions matérielles, les sentiments personnels, le domaine des études, etc. Ils devaient alors identifier les défis qu'ils et elles avaient eu à relever. Si leur arrivée était à refaire, que feraient-ils différemment? Quels conseils pourraient-ils offrir à leurs pairs? L'entretien les amenait à réfléchir à la construction d'un cours destiné aux étudiantes et étudiants étrangers : «Si on vous proposait un cours universitaire à votre arrivée en tant qu'étudiante ou étudiant étranger, quels éléments souhaiteriez-vous que ce cours inclue?» Après avoir exposé leurs idées, ils avaient à se prononcer sur les axes suivants : les interventions et les outils pédagogiques; le processus d'apprentissage; et les notions d'identité, d'appartenance et d'engagement dans le milieu universitaire. Leurs rétroactions ont été prises en compte dans l'élaboration du cours.

\section{STRUCTURE ET CONTENU DU COURS}

La démarche décrite ci-dessus a permis d'aboutir à la proposition du cours, dont l'objectif est de faciliter l'insertion universitaire des étudiantes et étudiants ayant réalisé leurs études antérieures dans des contextes culturels et éducatifs non canadiens, en les familiarisant avec la culture universitaire en milieu francophone nord-américain et en tenant compte du contexte social dans lequel s'inscrit cette culture. Le cours a été conçu comme un dispositif susceptible de favoriser la prise en charge, par les étudiantes et étudiants étrangers, de leurs apprentissages au moyen d'une réflexion approfondie sur 
les règles et les codes relatifs aux études universitaires qui est nécessaire à la réussite de leurs études.

Le cours se divise en trois volets : les interventions pédagogiques à l'Université Laval, le processus d'apprentissage et la réflexion sur l'appartenance, l'identité et l'engagement dans la vie étudiante. Cette structure est en partie inspirée des trois temps identifiés par Coulon. Le premier volet répond à la nécessité de connaître l'environnement que représente l'institution universitaire en milieu francophone nord-américain et donc de contrer le sentiment d'étrangeté. Au début du cours, le contenu de ce volet propose de se pencher sur les outils pédagogiques incontournables dans cet environnement, tels le règlement des études, le système de notation, le suivi du calendrier universitaire, le plan de cours (et sa fonction), les modes d'évaluation et le travail universitaire sollicité, ainsi que la connaissance des ressources qui sont à leur disposition (salles informatiques, bibliothèque, bureau de la vie étudiante, etc.).

En relation avec le temps de l'apprentissage évoqué par Coulon, les contenus du second volet se centrent sur les processus d'appropriation des savoirs. Ce volet aborde la notion du rapport aux savoirs et le concept de contrat didactique. Il propose des stratégies d'apprentissage et se penche aussi sur des questions de l'ordre des représentations sociales et des stéréotypes. D'après les travaux réalisés sous l'angle interculturel, ces questions touchent la facilité ou la difficulté des étudiants à s'insérer dans la vie sociale et universitaire.

Le troisième volet englobe les idées d'identité, d'appartenance et d'engagement dans le milieu universitaire. Cette étape peut paraitre prématurée, selon les "temps" proposés par Coulon, puisque ce type d'affiliation se construit au-delà de la première année universitaire, mais l'intention du cours est de l'approcher, de faire prendre conscience aux étudiants et étudiantes de l'importance du sentiment d'appartenance. Les contenus liés à ce troisième volet incluent la construction identitaire étudiante à partir de lectures et d'activités autour de l'histoire de l'Université Laval, du modèle des universités nord-américaines et du parler québécois. En prolongement de la connaissance du milieu offerte au premier volet, qui se limite aux outils pédagogiques, le contenu de ce troisième volet cherche à arrimer les notions d'engagement et d'identité étudiante en vue de l'acquisition, dans un plus long terme, du sentiment d'affiliation. 


\section{DE QUELQUES ASPECTS PÉDAGOGIQUES}

Les formules pédagogiques et les activités d'apprentissage retenues pour ce cours cherchent à favoriser l'expression personnelle et la communication interculturelle en créant une atmosphère de confiance et de réciprocité. En même temps, le cours se veut un espace d'expérience semblable aux autres cours (travaux à rendre selon un calendrier fixé dans le plan de cours, exigence de l'utilisation de la langue française, travaux individuels et d'équipe, présentations orales et travaux écrits, respect des consignes, etc.) afin de permettre aux étudiants et étudiantes de l'expérimenter et d'en parler (quels obstacles rencontrent-ils à la réalisation de travaux, comment vivent-ils le calendrier scolaire, le rythme des études, l'exigence des critères d'évaluations, etc. ?).

Selon une approche interactive, des ateliers d'exercices pratiques sont mis en œuvre en classe. Les mises en situation, les présentations par équipe et les discussions de groupe sont favorisées. Parmi les activités pédagogiques réalisées, je voudrais en présenter trois qui, à mon avis et par les commentaires reçus par les étudiants et étudiantes, le méritent. La première convoque des éléments identitaires en demandant une «mise en récit de soi ${ }^{42}$ ». La deuxième se démarque par son caractère interactif et sa pédagogie active. La troisième, utilisée comme activité d'évaluation, montre que ce processus est une occasion de poursuivre les apprentissages.

Premièrement, l'exercice de présentation de soi, dans la première phase du cours, cherche à mettre au jour les éléments biographiques qui distinguent chaque étudiant et étudiante. Il s'agit d'un instrument de réflexion les conduisant à expliciter les façons dont ils et elles ont décidé de faire des études à l'étranger, de venir au Canada, à Québec et, plus spécifiquement, à l'Université Laval. Le but n'est pas seulement de produire une description de leur cheminement étudiant, mais aussi de leur donner l'occasion de réfléchir sur une variété d'enjeux ayant marqué ce cheminement. En effet, les pratiques narratives en tant qu'outils pédagogiques peuvent constituer un outil privilégié pour les apprentissages de type réflexif ${ }^{43}$.

$$
\div+\div
$$

42. Selon une méthodologie inspirée des récits de vie et, plus largement, selon une approche narrative dont Ivor Goodson est l'un des représentants (Christophe NIEWIADOMSKY et Christine DELORYMOMBERger (dir.), La mise en récit de soi. Place de la recherche biographique dans les sciences humaines et sociales, Villeneuve d'Ascq, Presses universitaires du Septentrion, 2013 et Ivor GOODSON et Scherto GILL, Narrative Pedagogy. Life History and Learning, New York, Peter Lang, 2011).

43. Marie-Claude BERNARD, "Récits de pratique. Cas de figures en pédagogie universitaire ", Chemins de formation, $\mathrm{n}^{\circ} 19,2015$, p. 231-240. 
Par la suite, ce premier travail est bonifié par un récit de parcours dans lequel les étudiants et étudiantes présentent les raisons de leur choix de programme d'études et réfléchissent sur leur expérience en tant qu'anciens élèves et sur leur cheminement dans l'apprentissage. Cet exercice leur donne l'occasion d'analyser leur rapport aux savoirs et de réaliser une réflexion sur la diversité des situations d'apprentissage. Dans la mise en commun de ce travail, les notions d'identité étudiante et d'appartenance à un groupe sont proposées afin d'élargir leur réflexion vers le sens de l'engagement dans le parcours étudiant.

Deuxièmement, une activité particulièrement appréciée des étudiants et étudiantes était inspirée de dynamiques de groupe inscrites dans le domaine de la formation au dialogue communautaire ${ }^{44}$. Nommée «aquarium ", la formule pédagogique de cette activité consiste à placer les chaises de la classe en créant deux cercles (le premier, plus petit, au centre, le second, plus large, autour du premier). Le cercle intérieur représente l'«aquarium»; les personnes placées dans le cercle extérieur peuvent observer et écouter celles qui se trouvent assises au centre, participant, à cette première étape, de façon "passive". C'est sous cette forme que le thème des examens a été abordé. Invités au cours, des étudiants et étudiantes d'origine étrangère en fin de parcours universitaire et réussissant dans leurs études prirent place dans le cercle intérieur. Des questions sur les examens leur ont été proposées en guise de fil directeur, puis ils en discutèrent entre eux (par exemple, en parlant des différents types d'examens qu'ils avaient eu à passer, des éléments qu'ils avaient trouvés déroutants en comparaison de leur système scolaire antérieur, des actions à poser lorsqu'on échoue à un examen ou de celles qu'on devrait éviter, ou encore en donnant des conseils pour préparer les examens ou pour interpréter les consignes). Ils racontèrent aussi des anecdotes autour de la même thématique. Les personnes assises dans le cercle extérieur (les étudiantes et étudiants étrangers inscrits au cours) étaient très attentives aux propos de leurs pairs. Une fois que ces derniers ont épuisé le sujet, les étudiantes et étudiants du cours pouvaient leur poser des questions, et le dialogue s'est alors établi entre les participants et participantes des deux cercles. Dans les rétroactions faites pour le cours, les étudiantes et étudiants étrangers conseillèrent de refaire cette activité chaque session.

$$
\div+
$$

44. Ces pratiques s'inscrivent dans le sillon de la pédagogie dite "critique et émancipatrice» de Paulo Freire (Paulo FreIre, Pedagogía del oprimido, México, Siglo veintiuno editores, 1975). 
Finalement, je voudrais décrire une activité d'évaluation consistant en un travail d'analyse d'un récit véhiculant des notions étudiées en cours. Cette analyse cherche à la fois à mobiliser les acquis du cours et à permettre de faire ressortir la diversité des cheminements et l'identification de certains ancrages culturels. Le travail consistait à analyser les propos tenus par une Nigériane ayant fait ses études universitaires aux États-Unis. Celle-ci se présente en tant que "conteuse» dans une conférence filmée accessible en ligne avec sous-titrage et transcription intégrale en plusieurs langues ${ }^{45}$. Ses propos mettent en garde contre les discours dominants ou, selon ses propres mots, contre «le danger de l'histoire unique ». Un des enjeux majeurs de cet exercice était de faire une lecture critique des stéréotypes et des représentations sociales et de découvrir l'intérêt de la formation au langage interculturel.

En somme, le cours «Insertion universitaire en milieu francophone nord-américain ", offert par la DGPC, est à l'horaire à l'automne et à l'hiver depuis l'automne 2010. Les évaluations du cours réalisées par les étudiants et étudiantes qui l'ont suivi sont très encourageantes. Ceux-ci soulignent qu'il s'agit d'un cours qui permet de «comprendre et de s'adapter plus rapidement au nouveau milieu de vie ", qu'il «favorise la réussite ", qu'il "aide à mieux s'intégrer dans la vie universitaire». Ils remercient chaleureusement les enseignantes et certains affirment: «j'ai appris des choses que j'applique quotidiennement, et sincèrement, je constate les résultats positifs». Par contre, la pertinence du cours pour ceux et celles qui ne sont pas à leur première année à l'Université Laval est remise en cause : " Je m'interroge sur le côté obligatoire du cours pour des élèves déjà familiers avec la culture et la vie québécoises", certains sujets "n'étaient pas si importants pour moi [vu que j'étais déjà à l'université], mais je pense que c'est très utile pour les étudiants qui se trouvent dans leur première année à l'universitét ${ }^{46} \%$.

\section{CONCLUSION}

Le contexte de mobilité internationale et l'accès massif aux savoirs à travers le Web 2.0 pourraient porter à croire que notre monde progresse naturellement vers la construction d'une société plus équitable, plus participative

$$
\div+\div
$$

45. Chimamanda Ngozi ADICHIE, The Danger of a Single Story, conférence présentée dans le cadre de TED Global Conference, Oxford, juillet 2009, http://www.ted.com/talks/lang/fre_fr/chimamanda_ adichie_the_danger_of_a_single_story.html (30 août 2010).

46. Commentaires tirés des réponses des étudiants et étudiantes à l'évaluation du cours de l'automne 2010 et de l'hiver 2011. 
et plus «cultivée» (dans le sens de «littératie»). Cependant, cette vaste circulation des savoirs ne peut faire l'abstraction de l'émergence de nouvelles inégalités et de nouvelles formes de marginalisation, y compris en milieu universitaire. Les conditions de ce déploiement méritent qu'on s'y attarde. Comment éviter que les étudiants et étudiantes d'origine étrangère rencontrent l'échec? Autant la recherche documentaire que les entrevues réalisées dans le processus d'élaboration du cours montrent que ce qui est en jeu pour ces personnes n'est pas seulement de l'ordre des compétences cognitives, mais aussi de leur capacité de s'adapter aux «allants de soi », aux «nondits» de l'institution ${ }^{47}$. L'explicitation des outils pédagogiques et des attentes institutionnelles dans le premier volet du cours semble répondre au besoin de comprendre les «ficelles» d'un métier d'étudiant qui ne va pas de soi.

L'insertion réussie des étudiants et étudiantes d'origine étrangère ne peut pas faire l'abstraction du processus d'apprentissage, de l'appropriation des savoirs. Dans l'interaction nécessaire aux actes qui conduisent à l'apprendre, les étudiantes et étudiants étrangers doivent être outillés afin de développer des compétences qui ne sont pas forcément acquises au préalable. La conceptualisation exigée par les études universitaires demande également le développement de compétences de lecture, d'analyse critique et l'exercice de la réflexivité. À ces compétences s'ajoutent celles de savoir travailler en équipe et de faire des présentations orales, compétences qui sont favorisées par le référentiel de certains systèmes éducatifs plus que d'autres.

En ce qui concerne les résultats des travaux des étudiants et étudiantes ayant suivi le cours, ils ne sont pas uniformes. La pensée réflexive, l'analyse de leur propre rapport aux savoirs et à l'apprendre et le regard sur soi que demandaient certains exercices étaient plus approfondis chez les uns que chez les autres. L'intérêt pour la démarche réflexive exigée dans l'élaboration d'un récit de soi, par exemple, était manifeste chez certains et beaucoup moins chez d'autres. Le processus d'apprentissage et la construction des rapports aux savoirs étant caractérisés par un parcours individuel ${ }^{48}$, l'appropriation des savoirs témoigne aussi d'un parcours distinct.

$$
4+4
$$

47. Séraphin AlaVA et Marc RomainVILLE, "Les pratiques d'étude, entre socialisation et cognition", Revue française de pédagogie, $\mathrm{n}^{\circ} 136,2001$, p. 159-180.

48. Michel CAILlOT, "Les rapports aux savoirs des élèves et des enseignants ", Marie-Claude BERNARD, Annie SAVARD et Chantale BEAUCHER (dir.), Rapports aux savoirs. Clé pour analyser les épistémologies enseignantes et les pratiques de classe, 2014, p.7-18, http://lel.crires.ulaval.ca/public/le_rapport_aux_ savoirs.pdf (5 mars 2014). 
S'ajoute à ces compétences (qui ne sont pas toutes évoquées ici) la littératie numérique ou médiatique ${ }^{49}$, laquelle est considérée par certains comme un socle nécessaire à l'inclusion ${ }^{50}$. Cette littératie ne se limite pas à l'accès aux connaissances ni aux questions techniques ou procédurales, elle cherche aussi à intégrer la réflexion sur des enjeux sociaux et, plus particulièrement, politiques, économiques et éthiques, liés aux nouvelles technologies et à la circulation des savoirs qu'ils mobilisent ${ }^{51}$. Des voies restent à explorer pour intégrer les différentes littératies sollicitées dans le contexte mouvant de la circulation des savoirs qui caractérise les sociétés contemporaines. L'intégration de cette littératie nécessite des approches interactives, la création d'espaces de dialogue empathique, la mise en œuvre de communautés de pratique et l'intervention de tuteurs, de médiateurs qui favorisent les apprentissages ${ }^{52}$.

La question du langage interculturel est une autre clé essentielle à l'intégration des étudiantes et étudiants étrangers. Comme le soulignent les travaux de Castellotti et Moore, de Dervin ou encore de Py dans le domaine de la linguistique, les représentations sociales et les stéréotypes culturels jouent un rôle important dans les apprentissages et dans l'intégration ${ }^{53}$; dans certains cas, ils peuvent entraver le dialogue et faire obstacle à la compréhension mutuelle. Selon une perspective interactionniste, nos représentations et nos interprétations de ce que l' "autre» dit ou pense peuvent être source

$$
4+4
$$

49. Monique Lebrun, Nathalie LACELLE et Jean-François BOUTIN, "La littératie médiatique à l'école. Une (r)évolution multimodale, Globe. Revue internationale d'études québécoises, vol. 16, nº 1, 2013, p. 71 89.

50. CONSEIL NATIONAL DU NUMÉRIQUE, «Citoyens d'une société numérique. Accès, littératie, médiations, pouvoir d'agir. Pour une nouvelle politique d'inclusion ", rapport à la ministre déléguée chargée des petites et moyennes entreprises, de l'Innovation et de l'Économie numérique, http://www. cnnumerique.fr/inclusion (9 mars 2014).

51. Voir notamment la thèse de Patrick Plante (Patrick PLANTE, «Pour une problématisation de la technologie en éducation. Propositions théoriques pour un espace pédagogique alternatif de la technologie ", thèse de doctorat (technologie éducative), Québec, Université Laval, 2014).

52. En ce sens, la formule à distance offerte à partir de l'hiver 2015 mériterait une révision, car elle n'emploie pas le même espace d'interactions. Cette révision est d'autant plus nécessaire si l'on tient compte des difficultés éprouvées par certains des étudiantes et étudiants étrangers dans l'utilisation d'outils et d'un environnement numériques avec lesquels ils ne sont pas familiarisés.

53. Le travail de Véronique Castellotti et Danièle Moore, qui s'intéressent aux représentations sociales des langues, montre l'importance des représentations sociales des locuteurs sur les langues et leurs statuts visà-vis d'autres langues sur les stratégies mises en œuvre pour les apprendre et les utiliser (Véronique CASTELLOTTI et Danièle MOORE, "Représentations sociales des langues et enseignements ", Guide pour l'élaboration des politiques linguistiques éducatives en Europe. De la diversité linguistique à l'éducation plurilingue, Strasbourg, Conseil de l'Europe, 2002). L'étude de Fred Dervin sur les représentations du temps chez des étudiants français en programme d'échange international en Finlande est utile pour animer le débat des représentations et des stéréotypes (Fred DERVIN, "Temps et identifications vagabondes. Le cas des étudiants européens en Finlande», Migrations Société, vol. 19, n 110, 2007, p. 1-16). Le travail de Bernard Py, lui, évoque des obstacles (dus au langage) à l'intercompréhension (Bernard PY, "Une Mexicaine à Neuchâtel ", Bulletin suisse de linguistique appliquée, $\mathrm{n}^{\circ}$ 71, 2000, p. 71-85). 
d'incompréhension et de malentendus. Les compétences langagières et interculturelles peuvent être développées ${ }^{54}$ en favorisant des stratégies pédagogiques appropriées. Celles-ci incluent la déconstruction des stéréotypes et de certains clivages tels que celui des rapports dits "Nord/Sud " $" 55$ par le truchement de l'apprentissage mutuel, de la coopération et de la réciprocité.

Dans la visée d'une intégration réussie, ces aspects sont incontournables et peuvent s'arrimer au processus d'affiliation décrit par Coulon. L'affiliation n'est qu'embryonnaire à l'arrivée des étudiantes et étudiants étrangers et demande à ce qu'un suivi soit mis en ouvre. En effet, le dispositif dont il a été question ici montre l'intérêt des mesures à prendre pour l'accueil et l'intégration des étudiantes et étudiants étrangers, mais ces mesures ne peuvent se limiter aux étudiants d'origine étrangère de premier cycle ni à leur première session. La réflexion s'impose à l'université dans son ensemble si elle souhaite contribuer à l'édification d'une société du savoir inclusive.

54. Fred DERVIN et Elija SUOMELA-SALMI (dir.), Évaluer les compétences langagières..., op. cit., p. 115118.

55. Fran Martin et Helen Griffiths proposent pour cela une pédagogie socioculturelle (Fran MARTIN et Helen GRIfFITHS, «Power and Representation. A Postcolonial Reading of Global Partnerships and Teacher Development through North-South Study Visits ", British Educational Research Journal, vol. 38, $n^{\circ}$ 6, 2012, p. 907-927). 